\title{
Los sistemas intertextuales transmedia como estrategia pedagógica: De The Walking Dead® a La Odisea ${ }^{1}$
}

\author{
Recibido: 04 de diciembre de 2014 \\ Aceptado: 28 de mayo de 2015 \\ Publicado: 30 de octubre de 2015
}

\author{
Diego Montoya Bermúdez \\ dmonto36@eafit.edu.co
}

Miguel Arango Marín

marang44@eafit.edu.co

Universidad EAFIT (Colombia)

\begin{abstract}
Resumen: El presente artículo asume el estudio del fenómeno transmedia como un conjunto de relaciones intertextuales que involucra no sólo condiciones narrativas, sino también antropológicas, con dos objetivos: primero, el de estructurar un sistema intertextual que sirva de herramienta para el análisis de productos transmedia propios de la industria cultural; y segundo, el de generar claves posibles de ser usadas como estrategia pedagógica en el aula de clases para motivar la lectura y escritura en conjunto con otros sistemas simbólicos. Así, el artículo introduce al lector en el fenómeno de la transmedialidad, el cual es posteriormente revisado a partir de las propuestas de Henry Jenkins y Marsha Kinder que nos sirven como material para la elaboración de un Sistema Intertextual Transmedial, el cual se aplica como metodología para el análisis de un caso de la industria

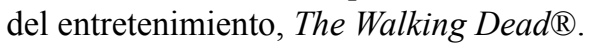

A partir de lo anterior y a manera de resultados, el texto identifica claves propias de este tipo de sistemas transmediales, las cuales son aplicadas como estrategias pedagógicas para la enseñanza en los niveles de educación básica secundaria y media en los colegios, como

\footnotetext{
1. Este texto presenta algunos resultados del proyecto de investigación "Aproximación a modelos de producción en proyectos transmedia aplicados a la educación y el entretenimiento desde el estudio a The Walking Dead®" asociado al trabajo de reflexión teórica desarrollado en el contexto del MediaLab y el semillero de creación hipermedia del pregrado en Comunicación Social de la Universidad EAFIT. Asimismo toma como referencia algunos puntos de la ponencia "La ficción zombi: una mirada transmedia", presentada en el XII Congreso ALAIC que aquí son desarrollados de una manera más profunda.
} 
se evidenciará en el estudio a La Odisea. Finalmente, a modo de conclusión expone la importancia de explorar las posibilidades que ofrece el estudio y la reflexión transmedial en ámbitos que trasciendan el mundo del entretenimiento.

Palabras clave: Narrativa transmedia, relaciones intertextuales, sistemas intertextuales transmedia, transmedia educativo, cultura de convergencia.

\begin{abstract}
This paper approach the study of transmedia phenomenon as a set of relationships that involves not only intertextual narrative conditions, but also anthropological. With two goals: first, to structure a system that serves intertextual analysis tool to own transmedia products of the culture industry and the second, to generate possible keys to be used as a pedagogical strategy in the classroom to encourage reading and writing in conjunction with other symbolic systems. So, the article introduces the reader to the phenomenon of transmediality, which is then reviewed proposals from Henry Jenkins and Marsha Kinder that serve as material for developing a transmedia Intertextual System, which applied as a methodology for the analysis of a case of the entertainment industry, The Walking Dead ${ }^{\circledR}$.

From the above and way of results, the text identifies key for this type of transmedia systems, which are applied as instructional strategies for teaching in basic education levels in secondary and high schools, as evidenced in the The Odyssey study. Finally, in conclusion exposes the importance of exploring the possibilities offered by the study and reflection transmedial in areas that transcend the world of entertainment.
\end{abstract}

Key words: Transmedia Storytelling, Intertextual Relationships, Transmedia Intertextual Systems, Transmedia Education, Convergence Culture.

\title{
1. Introducción
}

En el año 2003 el guionista de historietas Robert Kirkman, junto con Image Comics, revivieron el apocalíptico mundo de los muertos vivientes con el lanzamiento de The Walking Dead®. Ese mismo año, en una suerte de coincidencia, el investigador norteamericano Jenkins hablaría de "narrativa transmedia" como aquella posibilidad que ofrece la cultura de convergencia ${ }^{2}$ de expandir las historias a través de distintos medios. Una década después, ambos hechos terminaron imbricándose, en la medida en que el producto creado por Kirkman se convertía en uno de los fenómenos transmedia más exitosos para la industria del entretenimiento.

2. Jenkins (2008: 14) define convergencia como "el flujo de contenido a través de múltiples plataformas mediáticas y el comportamiento migratorio de las audiencias [...] dispuestas a ir casi a cualquier parte en busca del tipo deseado de experiencias de entretenimiento. 'Convergencia' es una palabra que logra describir los cambios tecnológicos, industriales, culturales y sociales en función de quienes hablen y de aquello a lo que crean estar refiriéndose". 
Actualmente, hablar de The Walking Dead® implica hacer alusión no sólo a las historietas, sino también a una serie para televisión, a webseries, videojuegos, novelas impresas, aplicaciones para dispositivos móviles, perfiles en Facebook, cuentas en Twitter, blogs, trailers, mashups, parodias, animaciones, performances, "escuelas para zombis" y hasta un escenario donde los fanáticos pueden sentir en carne propia lo que es ser un caminante de la muerte o un sobreviviente.

Como vemos, este caso de la industria del entretenimiento se tomó muy en serio el concepto acerca de lo transmedia, al punto que su análisis obliga a un abordaje no sólo narrativo, sino también antropológico (como lo propuso en su momento Scolari) en la medida en que en este fenómeno los usuarios (antes audiencias) asumen un papel fundamental para la expansión transmedia, que actualiza la propuesta hecha por Kinder (1991) al hablar de supersistemas comerciales de intertextualidad transmedia.

Desde este orden de ideas, el siguiente texto pretende, en primer lugar, presentar el concepto de sistema intertextual transmedia, como una propuesta que abarca las nociones de Kinder y Jenkins. En segundo lugar, a partir de dicha propuesta, analizar el fenómeno The Walking Dead ${ }^{\circledR}$ como un caso exitoso para la industria del entretenimiento transmedial. Finalmente y a manera de cierre, bosquejar una propuesta transmedial susceptible de ser aplicada en el ámbito educativo.

\section{Objetivos}

- Estructurar un sistema intertextual que sirva de herramienta para el análisis de productos transmedia propios de la industria cultural y de entretenimiento.

- Generar claves posibles de ser usadas como estrategia pedagógica en el aula de clases para motivar la lectura y escritura no sólo tradicional sino en conjunto con otros sistemas simbólicos.

\section{Metodología}

Para llevar a cabo el análisis a los productos que componen la ficción de la serie The Walking Dead, partiremos del análisis al concepto de universo narrativo que hacen Klastrup y Tosca (2004) desde la identificación el Mythos, Topos y Ethos (figura 1); conceptos que permitirán instalar el núcleo central narrativo que posibilita la elaboración de relatos derivados.

Identificado el universo narrativo (y para llevar a cabo el análisis a los relatos principales y derivados en The Walking Dead) tomaremos como referencia la propuesta metodológica que hacen Hernández y Grandio (2011), quienes partiendo de las ideas de Beeson (2005), Davidson (2008) y Scolari (2009), asumen el análisis a los relatos transmediales desde su relación con el medio en el que se instalan, los aspectos que configuran dicha narración, las capacidades de intertextualidad del relato central o principal con los relatos derivados y las formas como impactan en los usuarios. 
Figura 1: Metodología de análisis para los productos transmedia.

\begin{tabular}{|c|c|c|c|}
\hline \multicolumn{2}{|c|}{ CATEGORÍAS } & SUBCATEGORÍAS & DEFINICIÓN \\
\hline \multirow{3}{*}{ 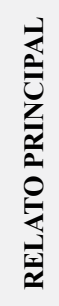 } & \multirow{3}{*}{ 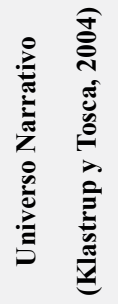 } & Mythos & Conflictos, personajes, criaturas, historias. \\
\hline & & Topos & $\begin{array}{l}\text { Periodo histórico (línea de tiempo), geografía, lenguaje, } \\
\text { poesía. }\end{array}$ \\
\hline & & Ethos & Ética y códigos de comportamiento. \\
\hline \multirow{5}{*}{ 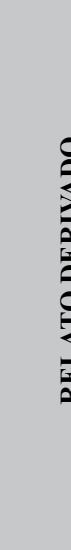 } & \multirow{5}{*}{ 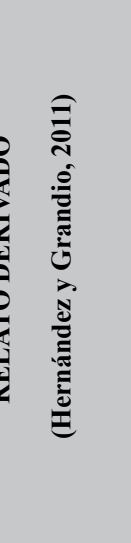 } & Relato & $\begin{array}{l}\text { Exploración del lenguaje utilizado en el medio o formato del } \\
\text { relato derivado. }\end{array}$ \\
\hline & & Medio & Plataforma de realización y distribución. \\
\hline & & Aspectos narrativos & Análisis del argumento, personajes, ambientación y tema. \\
\hline & & Intertextualidad & $\begin{array}{l}\text { Conexiones narrativas entre el producto original y el } \\
\text { derivado. Pistas de migración o "agujeros de conejo". }\end{array}$ \\
\hline & & $\begin{array}{l}\text { Distribución y } \\
\text { accesibilidad }\end{array}$ & $\begin{array}{l}\text { Niveles de implicación e interacción entre la narrativa } \\
\text { derivada y sus receptores (nueva creación, remix, creación } \\
\text { paralela). }\end{array}$ \\
\hline
\end{tabular}

Fuente: Elaboración propia, basada en Klastrup y Tosca (2004) y Hernández y Grandio (2011).

\section{Sistema intertextual transmedia (SIT)}

Cuando nos enfrentamos al fenómeno transmedia, según lo define Pratten (2011: 1) como el hecho de "contar una historia a través de múltiples medios de comunicación", nos encontramos ante una mirada netamente narrativa. No obstante, y cuando aparecen casos

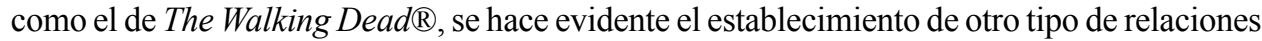
de orden paratextual, que involucra vínculos con medios, lenguajes, plataformas y modos de producción cultural que sobrepasan la noción tradicional sobre la narrativa. Es el caso de juguetes, productos de mercadeo, videojuegos, juegos de realidad alternativa y acciones en redes sociales, además de muchas otras prácticas que fijan actividades y producciones de los fans y usuarios como parte constitutiva de un sistema de entretenimiento complejo y diverso.

Así, se valida la idea de "sistemas intertextuales transmedia" (como parece sugerir Kinder y no únicamente de "narrativa transmedia") pues si bien la narratividad es una propiedad básica de este tipo de producciones, esta no resuelve la diversidad de productos 
y experiencias estéticas que proporciona. Kinder (1991: 42) destaca las relaciones de intertextualidad evidentes en los contenidos que producía la industria del entretenimiento a principios de los años noventa y como estos (los contenidos) eran presentados en distintos medios. A este fenómeno la investigadora lo denominó "supersistema comercial de intertextualidad transmedia".

El planteamiento de Kinder sugiere diferenciar los conceptos de "intertextualidad" y "transmedialidad" para unificarlos en una noción más completa. En ese sentido, asume la intertextualidad, leyendo a John Fiske, como la idea de que "cualquier texto es necesariamente leído en relación con otros textos" (ibídem: 46$)^{3}$, la cual se presenta en dos ejes: uno horizontal (que genera la relación entre los textos narrativos) y otro vertical, que involucra la relación entre un texto narrativo y otro tipo de textos (noticias, publicidades, críticas) y que ayudan a leer los primeros (ibídem: 46-47)4. Por otra parte y con relación al concepto de transmedialidad, esta fue asumida como la opción de tránsito que tiene una narración a través de distintos medios.

En ese orden de ideas, la noción de intertextualidad transmedia es crucial para Kinder en tanto "facilita no sólo la comprensión y el recuerdo de las historias, sino también el desarrollo de esquemas complejos de historias que se diferencian en los conflictos, personajes y modos de producción de la imagen" (ibídem: 59) 5 .

El panorama propuesto por Kinder nos resulta pertinente en el actual contexto de la cultura de convergencia, porque no limita el fenómeno transmedial únicamente a los aspectos narrativos. De este modo, asumimos como concepto macro el de sistema intertextual transmedia (SIT), entendido como una matriz de contenidos narrativos y paratextuales que se relacionan entre sí. Dicha matriz está conformada por los dos ejes arriba mencionados: $a$ ) el horizontal, eje al que hemos denominado diegético; y b) el vertical, que hemos planteado como eje paratextual ${ }^{6}$ (figura 2 ), el cual hace referencia a las producciones ubicadas en el eje diegético, pero que no constituyen una ampliación de la narrativa.

3. "The theory of intertextuality proposes that any one text is necessarily read in relationship to others" (texto original).

4. "[...] the distinction Fiske makes between horizontal forms of intertextuality (among primary texts) organized around genre or character, on the one hand, and vertical intertextuality 'between a primary text, such as a television program or series, and other texts of a different type that refer explicitly to it' (such as publicity, station ID's, journalistic articles, and criticism), on the other)" (texto original).

5. "[...] helps to facilitate not only the comprehension and recall of stories, but also the development of more complex schemata of what stories are like, with their highly complex patchwork of similarities and differences in plots, characters, iconography, mise-en-scène, and modes of image production" (texto original).

6. El concepto de paratextual lo asumimos a partir de Genette (1989), quien lo explica como: "títulos, subtítulos, prefacios, epílogos, advertencias, prólogos, etc.; notas al margen, a pie de página; epígrafes; ilustraciones [...] y muchos otros tipos de señales accesorias, autógrafas o alógrafas, que procuran un entorno (variable) al texto". El concepto de Genette nos permite pensar la narrativa transmedia (en el eje diegético) como analogía de la obra literaria y los demás productos que no tienen injerencia en el desarrollo de la narrativa, y también, por extensión análoga, como sus paratextos integrados al sistema intertextual transmediático. 
Figura 2: Sistema intertextual transmedia.

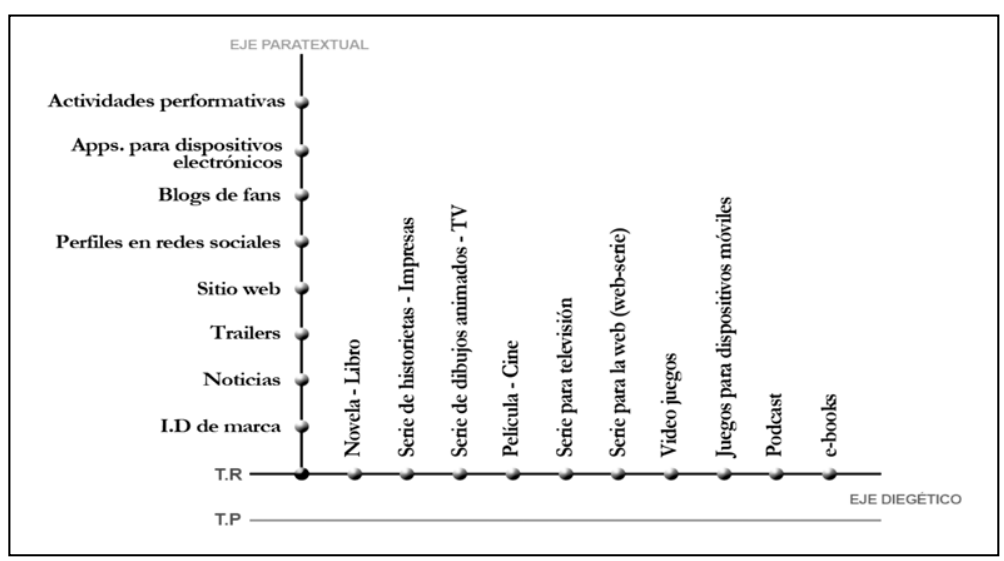

Fuente: Montoya, Vásquez y Salinas (2013: 148).

Como se observa en el gráfico anterior, la narrativa transmedia se encuentra constituida únicamente por aquellos productos que se ubican en el eje diegético, y al sumar ambos ejes lo que obtenemos es el sistema intertextual transmedia. Ahora bien, la propuesta de los SIT se hace potente en la medida en que no se limita sólo a la ubicación de los productos narrativos y paratextuales en la matriz del sistema, sino que también sirve como herramienta para el análisis del factor tiempo en las producciones diegéticas, por un lado, y por el otro para ubicar la obra que posibilita el fenómeno transmedia. Explicaremos estas tipologías en los siguientes subapartados.

\subsection{Tipología 1: tiempo del relato y tiempo de la producción}

Cuando nos encontramos ante la matriz de intertextualidad transmedia, además de los ejes vertical y horizontal, encontramos en este último una doble línea que nos presenta dos tipos de temporalidades diferentes: una superior que hemos denominado "tiempo del relato" (TR) y una inferior que llamamos "tiempo de la producción" (TP).

La línea superior nos ubica en el tiempo en el que está ambientada la historia, el cual en muchas oportunidades no es anunciado de forma literal, pero que puede ser inferido por el espectador a partir de los elementos que se encuentran en el mundo posible, los cuales pueden ser comparados con el mundo de referencia ${ }^{7}$. Ahora bien, el primer tiempo del relato está dado por la primera obra (obra seminal) y es este el que nos sirve para analizar las obras producidas con posterioridad, las cuales pueden tener tres posibilidades temporales diferentes con respecto a la obra seminal: anterior (precuelas), igual (sincrónicas) o posterior (secuelas).

7. Para ampliar las nociones de mundo posible y mundo de referencia, ver el capítulo 8 "Estructuras de mundos" de Lector in fábula (Eco, 1993), del cual tomamos dichos conceptos. 
En síntesis, el tiempo del relato hace referencia a todo el eje diegético y nos sirve para identificar en qué tiempos están contadas las producciones realizadas en cada formato después de la obra seminal (precuelas, sincrónicas o secuelas), independientemente de su fecha de realización; de esta forma, los tiempos del relato no tienen que ser cronológicamente secuenciales $^{8}$. Respecto a la línea inferior o tiempo de la producción, esta ubica el año de lanzamiento de cada obra incluida en el SIT y nos sirve como herramienta para ordenar la aparición de los productos en su secuencialidad cronológica.

\subsection{Tipología 2: obra seminal y obra núcleo}

Otro aspecto que se desprende del análisis de la matriz propuesta en el gráfico tiene que ver con el concepto de obra seminal, el cual hace alusión al producto que da inicio al sistema intertextual transmedia; aunque es necesario aclarar que la obra seminal no es la única que posee la condición de expandir una narrativa transmedia. Cualquier obra ( $\sin$ importar su formato) es potencialmente creadora de un SIT y cuando un producto se deriva de ella pueden ocurrir dos situaciones: que inicie un sistema transmedia o un sistema crossmedia. Este último caso se da gracias a que entre una obra y otra hubo adaptación en diferentes formatos. Ahora bien, cuando el fenómeno tiene como característica el que de una obra a otra haya una expansión, este se reconoce como un "sistema transmedia"; y a la obra que potencia el desarrollo de dicho sistema la denominamos "obra núcleo".

Entendemos entonces por obra núcleo aquella que permite la expansión del universo narrativo o paratextual. En ese orden de ideas, una obra seminal puede ser al mismo tiempo la obra núcleo que dé nacimiento a la narrativa transmedia. Pero, ¿cómo identificar que una obra es núcleo?

En el sistema intertextual transmedia, los productos que lo conforman se referencian y se interrelacionan entre sí en uno o en ambos ejes (figura 3); de este modo, el producto que mayores relaciones intertextuales genera, tanto en el eje diegético como en el eje paratextual, es el núcleo del sistema. Para visualizar esto nos parece pertinente acudir al caso que hemos elegido para el análisis de un SIT. Así, en el siguiente punto revisaremos el desarrollo de la matriz a la luz de The Walking Dead ${ }^{\circledR}$, en cuanto referente de transmedialidad en la industria del entretenimiento.

\section{Resultados}

\subsection{La matriz intertextual transmedia en el caso The Walking Dead® (TWD)}

TWD es una serie de televisión producida desde 2010 por el canal estadounidense AMC® y que transmite para Latinoamérica la cadena $F O X ®$. La serie narra la historia de un pequeño

8. No se deben confundir las "precuelas" y "secuelas" con las analepsis y las prolepsis. Las primeras comprenden las historias creadas a partir del tiempo anterior (precuelas) o posterior (secuelas) a la obra seminal, mientras que las segundas son los saltos en el tiempo hacia atrás o hacia adelante al interior de cada una de esas mismas historias. 
grupo de personas que escapan al apocalipsis zombi y que deambulan a través de EE. UU. en busca de un lugar seguro que los mantenga a salvo de las hordas de muertos vivientes.

Inicialmente la serie nace del cómic homónimo que en 2003 empieza a producir la empresa Image Comics ${ }^{\circledR}$ para el mercado norteamericano. En 2009, AMC ${ }^{\circledR}$ obtiene los derechos de la historia y anuncia la producción de una serie para televisión. Antes de darse este hecho y como forma de promoción, $A M C \circledR$ realiza una adaptación en dibujos animados de la primera historieta que es presentada en el Comic-con de 2010. Ese mismo año, para la celebración del día de Halloween se estrena la serie para la televisión mundial, respetando el comienzo de la historia del cómic impreso, aunque después es alterada al incluir nuevos personajes y modificar algunos hechos.

Cuatro años después del lanzamiento de la serie para televisión, TWD se mantiene como un caso exitoso de transmedialidad, en la medida que su universo narrativo ha posibilitado la creación de múltiples historias que han expandido el relato por diferentes medios, generando así la creación de un amplio grupo de fans en todo el mundo. A continuación, y para retornar al análisis de los SIT, veamos el comportamiento del caso de estudio en la figura 3 que corresponde a su matriz transmedia.

Figura 3: Sistema intertextual transmedia aplicado al caso The Walking Dead ${ }^{\circledR}$.

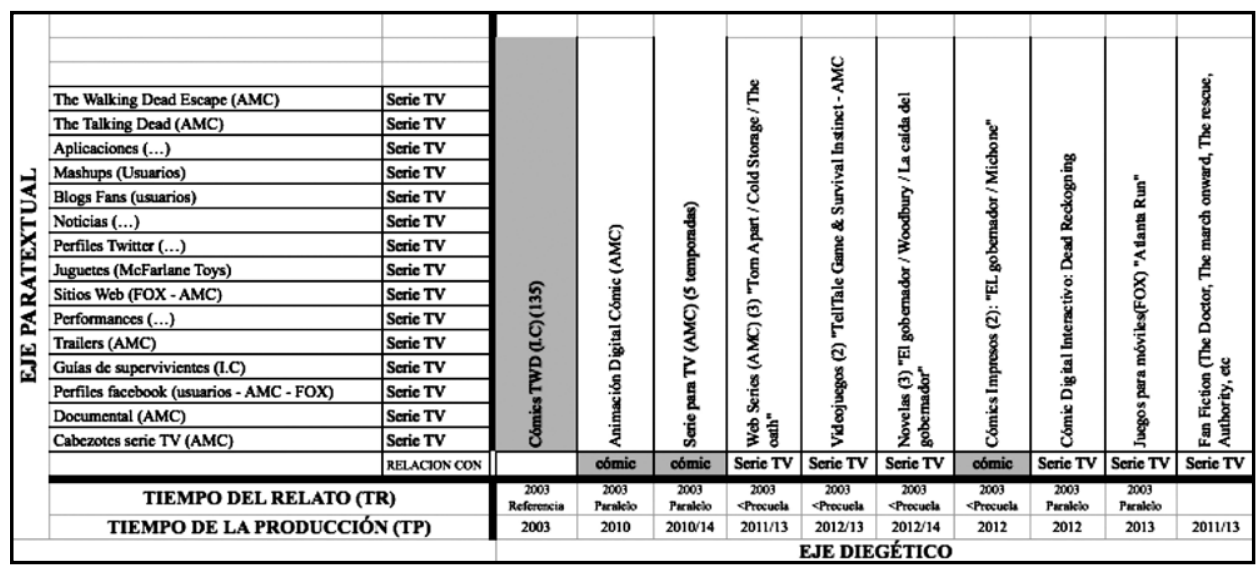

Fuente: Elaboración propia.

Como se puede apreciar, el caso The Walking Dead ${ }^{\circledR}$ muestra su alcance como sistema intertextual transmedia, aclarando obviamente que las obras que recogemos en la matriz son sólo una muestra de las más representativas para nuestro análisis; en ese sentido podemos decir que la potencialidad de crecimiento permanente que presenta la matriz nos permite reconocer la característica dinámica de los SIT. Una vez aclarado este punto, procedamos a analizar el caso.

Como ya lo explicamos, TWD nace en 2003 como una serie de cómics impresos de emisión mensual y que diez años más tarde continúa en un número que supera el centenar de entregas. 
Este producto (nos referimos a la historieta) es claramente identificable como obra seminal dentro de la matriz. Siete años después de publicada su primera edición, aparece el primer producto en un formato distinto, a saber, un episodio de dibujos animados realizado como adaptación el cual conservó los mismos personajes, lugares, hechos e ilustraciones del cómic. Hasta ese momento el sistema fue crossmedia.

El mismo año en que aparece el episodio de dibujos animados, la productora lanza al mercado mundial la serie de televisión, la cual conservó en su primer episodio lo que mostraba la primera historieta y el dibujo animado, por lo cual la adaptación se mantuvo. Pero a partir del segundo episodio las cosas empezaron a cambiar: aparecieron dos personajes nuevos para el universo narrativo que propuso TWD; Merle y Daryl Dixon, dos hermanos que por sus características de rudeza y de generación de conflicto fueron fundamentales para la evolución del relato. Después de esto, la serie de televisión cada vez se fue tomando licencias para generar nuevos sucesos, alterar otros existentes en el cómic, prescindir de personajes y crear otros.

En el receso entre la primera y segunda temporada (y con el fin de mantener la conexión de los fans al producto) AMC $\AA$ produce una serie-web conformada por seis webisodios (cada uno de ellos con una duración de entre 3 y 6 minutos) la cual contaba la historia de un personaje secundario que había aparecido en el cómic impreso, así como en la animación y en el primer episodio de la serie. Se trataba del primer muerto viviente que Rick Grimmes (el protagonista) se encuentra, y al que en un gesto de compasión elimina propinándole un disparo en la cabeza. La web serie se conoció como "Torn apart" y narra la historia de este personaje antes de haberse convertido en zombi y cómo llegó a ese estado. Es en ese momento cuando encontramos el primer caso de expansión producido en TWD, pues acá la historia (que era completamente nueva) conservaba las características propuestas en el universo narrativo instalado en el cómic y generaba una ampliación de la diégesis.

Después de este primer hecho expansivo en el plano de lo diegético, la característica se convirtió en una constante que dio como resultado la creación de nuevos personajes e historias en otra serie-web ("Cold Storage") en dos videojuegos para consolas, en dos novelas, entre otros productos. Precisamente en el segundo videojuego producido, la historia la protagonizan los hermanos Dixon, quienes en la serie eran personajes secundarios y ni siquiera existían en el cómic.

Ahora bien, cuando empezamos a observar la relación entre los distintos productos que aparecen en los ejes del SIT, nos encontramos con una característica: el capítulo de dibujos animados, el primer episodio de la serie y un par de cómics impresos sobre otros dos personajes de la historia original (El gobernador y Michonne) hacen referencia intertextual a la obra seminal. El resto de productos demuestran su relación intertextual con la serie, como en el caso del videojuego "Survival instinct" donde aparecen Merle y Daryl, personajes originarios del producto televisivo y no del cómic impreso; además, los modelos animados digitalmente en 3D están basados en las características físicas de los actores Norman Reedus (Daryl) y Michael Rooker (figura 4). 
Figura 4: Franquicia The Walking Dead®.

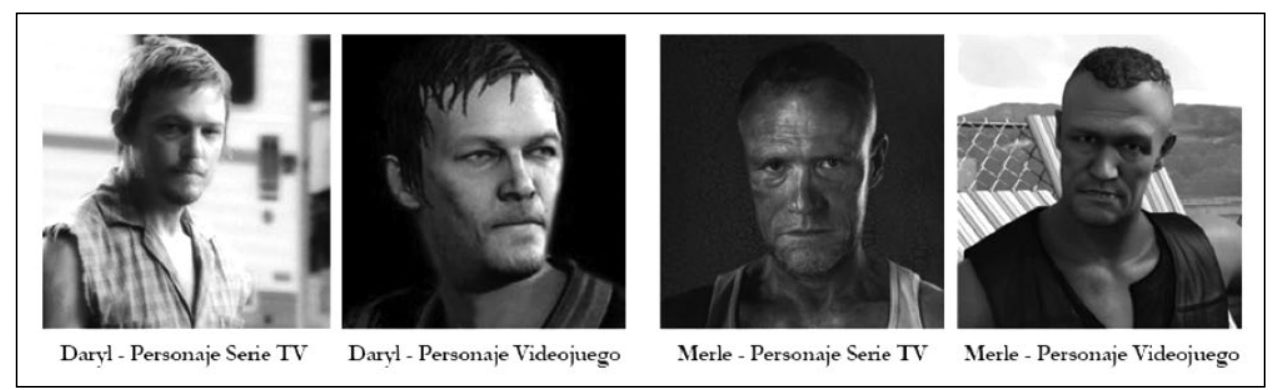

Fuente: Elaboración propia.

Otro caso de relación intertextual con la serie se dio con la aplicación para tablets de un formato cómic digital interactivo creado por AMC $($, y que tituló "Dead reckoning". Este producto, a pesar de utilizar los mismos personajes que aparecen en el cómic, cuenta una nueva historia y su vínculo con la serie de televisión (y no con la historieta) radica en que las ilustraciones conservan las características físicas de los actores de televisión (figura 5).

Figura 5: Franquicia The Walking Dead®.

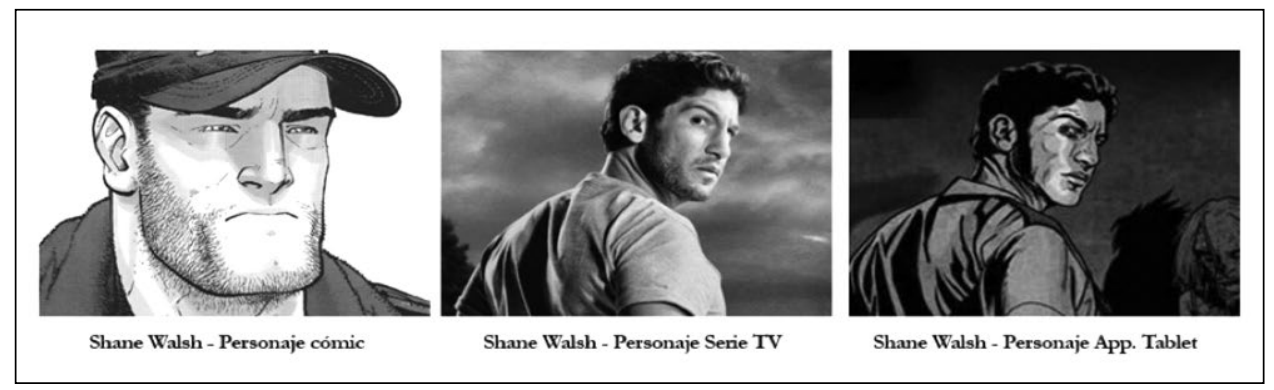

Fuente: Elaboración propia.

Pero estas relaciones intertextuales no sólo se dan entre obras que hacen parte del eje diegético. Como ya explicamos, las relaciones se pueden dar en cualquier vía, entre productos de uno o de ambos ejes. Un ejemplo lo encontramos en el personaje de Michonne, una abogada que cuando se ve enfrentada al apocalipsis zombi saca de sí las características de un sujeto frío, reservado y aguerrido.

Gracias a la aceptación de los fans hacia este personaje, AMC® junto con la empresa McFarlane Toys ${ }^{\circledR}$, deciden sacar una serie de muñecos articulados y Michonne es uno de ellos. Precisamente el juguete que la compañía produce se reconoce como relación intertextual con la serie en la medida en que el modelo conserva las características físicas de la actriz Danai Gurira quien encarna al personaje (figura 6). 
Figura 6: Franquicia The Walking Dead®.

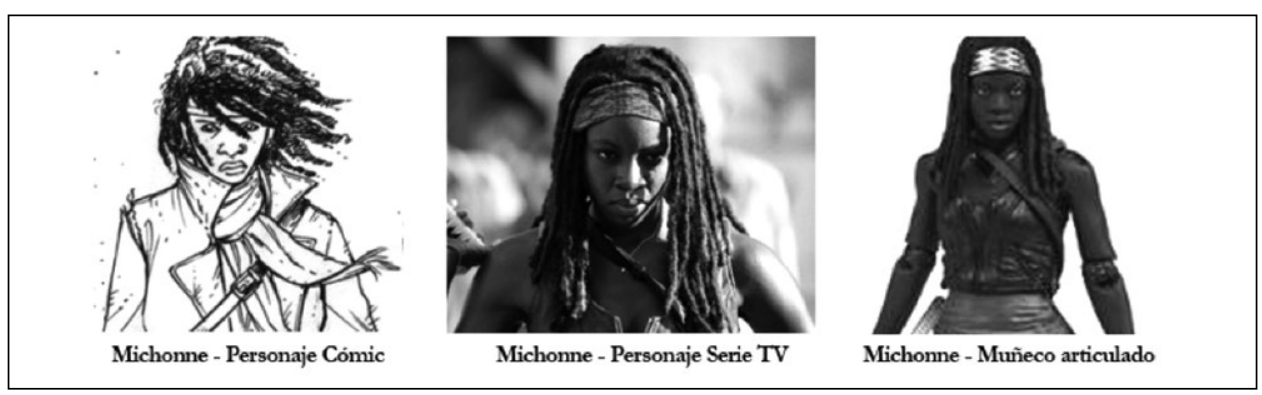

Fuente: Elaboración propia.

De este conjunto de relaciones intertextuales (de la cual señalamos únicamente tres productos entre un universo tan amplio como el que proporciona la web) que remiten a la serie de televisión, podemos concebir a esta como la obra núcleo del sistema.

En lo concerniente a la característica del tiempo evidenciada en el SIT de The Walking

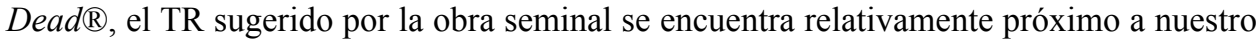
mundo de referencia. La constante en el TR es que las obras posteriores a la obra seminal (que bien pueden ser narradas como precuelas o secuelas) juegan con un tiempo histórico muy cercano al propuesto por la obra seminal. Es el caso del videojuego, producido por Telltale games ${ }^{\circledR}$, que comienza unos pocos días antes del sugerido en el primer cómic (y que por ende sería una precuela), pero el cual rápidamente se instala en un tiempo sincrónico al planteado en el cómic. Esto mismo sucede con el videojuego "Survival instinct", la serie-web "Torn apart” y la primera novela del gobernador.

En síntesis, el tiempo en el SIT de TWD se da de dos formas: las precuelas, cuyas creaciones no son muy numerosas, y las cuales se sincronizan rápidamente con la obra seminal; y las sincrónicas, que mantienen un mismo tiempo con dicha obra inicial. Estas son las que más se evidencian, pues cada una de ellas conserva el contexto instalado por el cómic y siempre se hace referencia al espacio en el que los humanos sobreviven a las hordas zombis. En el caso de las obras en clave de secuela, estas no se identificaron en el SIT.

\subsection{Hacia un modelo de sistema transmedia en otros ámbitos}

Como vemos, el potencial de un sistema intertextual transmedia es sumamente amplio, entre otras razones, porque nos permite: a) profundizar en las diferencias existentes entre el fenómeno transmedia y el crossmedia; $b$ ) entender, interpretar y analizar las claves temporales y las relaciones presentes entre los distintos textos que integran el sistema; y c) reconocer cuál es la obra núcleo, un asunto de suma importancia, puesto que permite identificar los intereses de los usuarios, los cuales participan de una manera activa en la construcción dinámica del sistema y porque dichos usuarios, dentro del contexto de la cultura de la convergencia, son una pieza clave para garantizar el éxito comercial de un proyecto transmedia. 
Ahora bien, con el análisis que hemos expuesto hasta aquí no pretendemos restringir las conclusiones de nuestra estudio al hecho de poner a prueba el sistema intertextual transmedia, ni mucho menos a llegar a la obvia reconfirmación del éxito que ha tenido en la industria del entretenimiento un caso de narración transmedial como The Walking $\operatorname{Dead}^{\circledR}$, sino más bien, poner de manifiesto que esta investigación nos ha conducido a la identificación de una serie de condiciones o claves propias de las narraciones transmediales que pueden ser retomadas para la construcción de modelos que son susceptibles de ser aplicados en ámbitos distintos al del entretenimiento.

\subsection{Claves para un modelo transmedial}

Si entendemos las narrativas transmedia como un sistema que no solo se configura a partir de un nivel narrativo (diegético), sino que además está siendo claramente afectado y conformado por una serie de fenómenos de recepción y apropiación individual y colectiva del sentido ${ }^{9}$ a partir de los consumos mediáticos ${ }^{10}$ (paratextual), se hace entonces necesario que presentemos aquellas condiciones o elementos claves para la estructuración de un modelo transmedial desde ambas perspectivas. De esta manera, comenzaremos con aquellos aspectos que, desde el punto de vista narrativo, son esenciales ${ }^{11}$ para el propósito que nos hemos trazado.

De esta forma, si queremos llegar a transmedializar un relato, debemos tener presente que este debe estar apoyado por un universo narrativo sólido que trascienda la trama general de la historia que se quiere contar, además, debe permitir que las posibles historias de la narración estén soportadas en diversas plataformas mediales. De igual forma, resulta relevante considerar que las narraciones transmediales cobran sentido y se legitiman principalmente cuando son acogidas por usuarios proactivos que contribuyen con sus iniciativas a la ampliación del universo narrativo.

A esto se suma la utilización de tres conceptos claves a saber: a) de los espacios en blanco, entendidos como aquellos puntos de indeterminación que quedan en todo texto y terminan

9. La ampliación de esta particularidad del eje paratextual se encuentra consignada en (Montoya, Vásquez y Salinas, (2013).

10. Para Appadurai (2001: 7), en un mundo globalizado y mercantilizado como en que nos encontramos, las relaciones sociales se configuran en buena medida en un proceso de "aclimatación" de productos de consumo mediáticos globales. Así, según el pensador hindú: "Debido a las pura multiplicidad de las formas que adoptan (el cine, la televisión, las computadoras) y a la velocidad con que avanzan y se instalan en las rutinas de la vida cotidiana, los medios de comunicación electrónicos proveen recursos y materia prima para hacer de la construcción de la imagen del yo, un proyecto social cotidiano".

11. Autores como Jenkins (2006), Long (2007) y Gallego (2011) se han ocupado de la reflexión sobre la narrativa transmedia y que han llegado en sus conclusiones a la identificación de puntos esenciales para la configuración de narraciones transmediales exitosas. Estos aportes los hemos considerado como referentes valiosos para la consolidación de nuestra propia propuesta. 
por ser generadores de ampliaciones diegéticas aprovechadas tanto por el autor como por los lectores de un relato; b) las señales de migración, descritas como pistas o cuestiones no resueltas que un autor deja en su relato para motivar la curiosidad de sus lectores y conducirlos a otras historias y plataformas mediales para resolverlos; y c) los agujeros de conejo, refiriéndonos a aquellas entradas a un mundo ficticio vinculado y activado desde los productos paratextuales utilizados por los usuarios para la ampliación del universo narrativo de un relato en particular ${ }^{12}$. Tres conceptos que, decíamos, son esenciales para la comprensión, expansión y experimentación inmersiva de una narración transmedial.

Ya desde un punto de vista paratextual, nos encontramos con que las claves para la estructuración del modelo transmedial que queremos son menos concretas que las del nivel diegético y han sido poco tratadas por los autores aquí expuestos ${ }^{13}$; pero consideramos que es en ellas donde radica la relevancia del sistema intertextual transmedia. Pues dichas claves nos abren la posibilidad de pensar el estudio de los fenómenos transmediales, no únicamente desde un punto de vista narratológico, sino también social y antropológico. Por lo tanto, podemos considerar que el proceso de la transmedialización de los relatos, puede trascender la narrativa del mundo del entretenimiento para ser aplicados en otras esferas socio-culturales.

Bajo estas circunstancias, mencionaremos dos de las condiciones claves para la generación de un modelo transmedial desde una perspectiva paratextual. La primera se basa en comprender los procesos de recepción y apropiación de un relato por parte de sus lectores como un modo particular para la generación del sentido y la identidad tanto individual como colectiva ${ }^{14}$. Con esto entendemos que desde el orden de lo paratextual es posible motivar la interrelación, la participación y la cooperación entre diversos grupos de lectores o usuarios alrededor de un universo narrativo dado. Relaciones que terminan favoreciendo la salud y el recuerdo del relato, pues cumplen la función fundamental de darles a los lectores la posibilidad de ampliar en su vida cotidiana la experiencia ofrecida por la narración.

La segunda condición consiste en saber que los procesos de apropiación de los contenidos mediáticos, por parte de los usuarios, pueden ser entendidos como una valiosa fuente de inspiración para la renovación creativa del componente propiamente narrativo, esto es, del nivel diegético del sistema transmedial. Esto último, genera una especie de co-creación entre el autor del texto y los lectores o usuarios del mismo.

12. Para la ampliación de estos conceptos ver: Umberto Eco (1993) en el caso de los espacios en blanco, Marc Ruppel (2006) citado por Long (2007) y Gallego (2011) para las señales de migración y McGonigal citada por Sternos, Monttola y Björk (2009) para los agujeros de conejo.

13. Con excepción de la ya citada Kinder.

14. Pensemos, por ejemplo, en cómo la recepción que ha tenido la temática zombi a nivel global ha posibilitado que miles de personas se apropien y se identifiquen profundamente con ella. Prueba de ello, pueden ser aquellos grupos que se reúnen en distintas partes del mundo para la realización de marchas donde cientos de fanáticos disfrazados de muertos vivientes caminan lentamente por las calles de las ciudades. Estos eventos vistos como fenómenos sociales, responden a lo que Varela (1999), apoyada en García Canclini, define como la construcción de la identidad individual y colectiva de manera fragmentada, atomizada y globalizada a partir de los consumos mediáticos contemporáneos. 


\subsection{Una propuesta aplicada al entorno educativo}

Luego de identificar las posibilidades que se abren al comprender los procesos de transmedialización más allá de su dimensión puramente narrativa, nos surgió la motivación de consolidar un modelo transmedial para aplicarlo en el entorno educativo. Con esto en mente, partimos de la idea de implementar un sistema intertextual transmedia en el aula de clase como complemento en las dinámicas de aprendizaje de la literatura clásica en los estudiantes de secundaria. Así, y bajo los Estándares Básicos de Competencias del Lenguaje propuestos por el Ministerio de Educación Nacional, específicamente en dos de sus tres campos ${ }^{15}$, nos dimos a la tarea de transmedializar La Odisea con el fin de incentivar en los estudiantes de bachillerato la lectura de clásicos de la literatura universal como lo propone el documento oficial:

"la pedagogía de la literatura obedece a la necesidad de consolidar una tradición lectora en las y los estudiantes a través de la generación de procesos sistemáticos que aporte al desarrollo del gusto por la lectura, es decir, al placer de leer poemas, novelas, cuentos otros productos de la creación literaria que llenen de significado la experiencia vital de los estudiantes y que, por otra parte, les permitan enriquecer su dimensión humana, su visión de mundo y su concepción social a través de la expresión propia, potenciada por la estética del lenguaje" (MEN, 2006: 25).

En Colombia, según la encuesta de consumo cultural realizada por el DANE (2013), nuestros estudiantes leen menos de dos libros por año, no obstante el consumo de cine, radio, televisión, videojuegos es alto. Este panorama nos demuestra por un lado, que se hace necesario incentivar la lectura tradicional y por el otro, que las demás formas de consumo cultural deben ser también consideradas como otro tipo de lectura, en tanto la idea de texto se puede asumir no sólo para lo escrito, sino también para lo sonoro y lo visual. En ese sentido, la idea de pensar la transmedialización de un clásico de la literatura como lo es La Odisea, permite, además de consolidar la tradición lectora que pide el Ministerio de Educación, ampliar las maneras en que este texto puede ser leído, en tanto un sistema intertextual transmedia en este contexto permite la creación de múltiples piezas del orden de lo sonoro, lo visual y lo cibermedial, cumpliendo además con otro de los objetivos planteados en el documento oficial, y es el de "estimular la capacidad productiva de los estudiantes, es decir, estimular y propiciar la escritura con intención literaria: cuentos, sociodramas, poemas, ensayos, etc. De tal manera que puedan expresar sus formas particulares de sentir, ver y recrear el mundo" (MEN, 2006: 26) y como complementa el documento, "formar en lenguaje implica avanzar también en el conocimiento de otros sistemas" como los gestuales, los sonoros, los visuales y los audiovisuales.

\footnotetext{
15. El documento Estándares Básicos de Competencias del Lenguaje propone el trabajo en tres campos: a) pedagogía de la literatura; b) pedagogía de otros sistemas simbólicos; y c) pedagogía de la lengua castellana. Para efectos de la investigación, decidimos no trabajar de manera explícita este último pues consideramos que la competencia comunicativa de los estudiantes, la cual se propone en este campo, se va a desarrollar de forma implícita en la medida en que la construcción del sistema transmedia requiere de la interrelación de estudiantes y profesores de distintas escuelas y grados.
} 
Con estos lineamientos oficiales, nos dimos a la tarea de construir un sistema intertextual transmedia de La Odisea (figura 7), a partir del trabajo de un equipo interdisciplinario ${ }^{16}$ conformado por estudiantes de bachillerato, estudiantes universitarios de carreras como la comunicación social, la publicidad, el diseño gráfico y la ingeniería, además de profesores competentes en literatura y en producción de otros sistemas simbólicos. En "Odisea, una narrativa transmedia" (2013), el texto original entraba al SIT con la doble característica de ser obra seminal y núcleo, en la medida en que todos los productos propuestos por los estudiantes (adaptaciones y expansiones) ampliaban el universo narrativo del texto a través de diversas historias soportadas en distintos $\operatorname{medios}^{17}$.

Figura 7: Sistema intertextual transmedia aplicado a La Odisea.

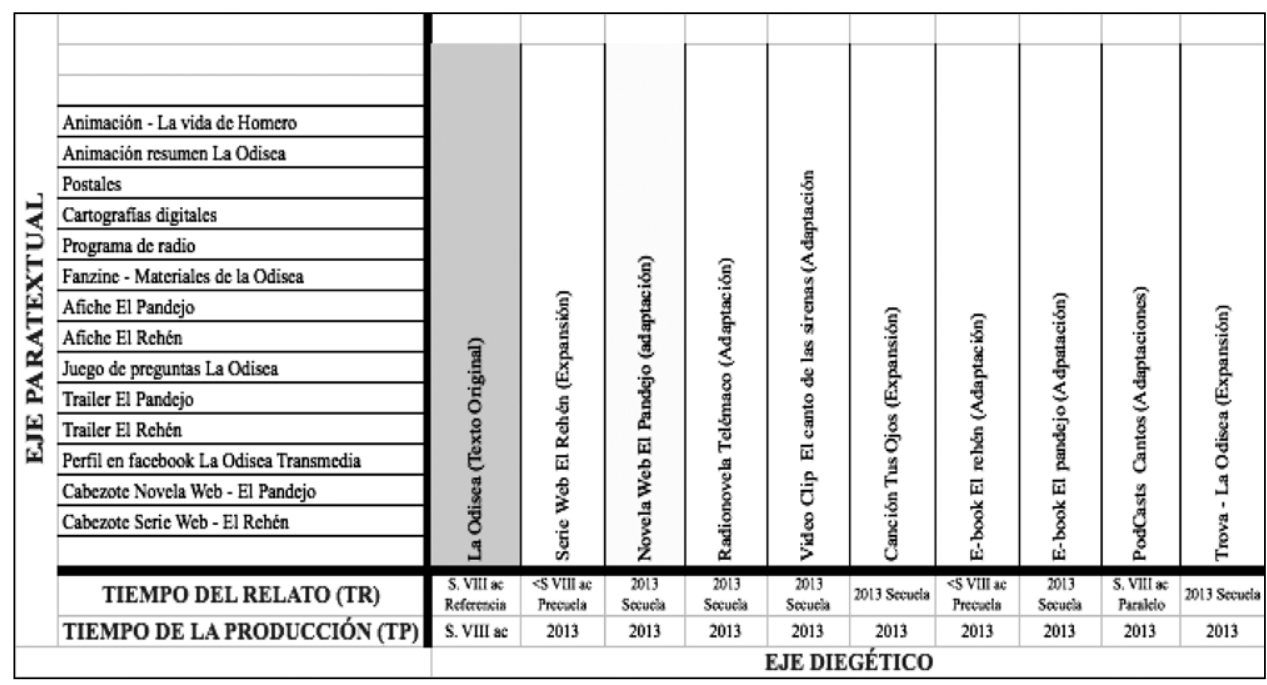

Fuente: Elaboración propia.

Al igual que sucede con el SIT de The Walking Dead®, este (refiriéndonos al aplicado a La Odisea) permite ver cómo la estrategia de transmedialización del clásico de la literatura generó un trabajo activo por parte de estudiantes y profesores, en la medida

16. La implementación del Sistema Intertextual Transmedia se llevó a cabo durante el segundo semestre de 2013 en el marco del Plan Digital TESO (2013), proyecto de la Alcaldía de Itagüí y la Universidad EAFIT y participaron 30 personas, entre estudiantes y profesores de las I. E. María Josefa Escobar y Pedro Estrada, además de estudiantes de Comunicación Social de la Universidad EAFIT y estudiantes de Diseño de la Universidad Pontificia Bolivariana y profesores de ambas universidades.

17. Algunos de los productos realizados fueron un fanzine con el mundo material de La Odisea, dos series web: una de ellas contextualizada en la época en que supuestamente vivió Homero y ficcionando una posible historia del autor y otra en formato telenovela haciendo una adaptación a los personajes en un contexto actual. Una radionovela adaptada en un contexto contemporáneo, cartografías, pósters, afiches, cómics y animaciones con adaptaciones a algunos cantos de La Odisea, entre otros productos. 
en que resultaron diversos productos (diegéticos y paratextuales) en torno al universo narrativo propuesto por La Odisea. Este hecho nos conecta nuevamente con los estándares propuestos por el Ministerio de Educación Nacional, cuando habla de "incentivar el gusto por la lectura [incidiendo así] en el desarrollo de competencias relacionadas con lo estético, lo emocional, lo cultural, lo ideológico, lo cognitivo y lo pragmático” (MEN, 2006: 25).

Es importante resaltar que el objetivo del proyecto no era nunca el de reemplazar la lectura del clásico, sino de motivar la misma para que los estudiantes después de hacerlo, generarán producciones en diversos sistemas simbólicos, los mismos que implican de base la lectura y escritura tradicional, por ejemplo cuando realizan los guiones para formatos audiovisuales. En ese sentido, el trabajo de transmedialización de La Odisea permitió proponer un esquema a partir de los estándares básicos de competencias de lenguaje del MEN, en el que se tuvieron en cuenta los campos pedagógicos y los identificadores tanto en la comprensión del texto como en la producción de sentido, además de la importancia de formar equipos interdisciplinarios al que denominamos citando a Lévy (2004) como colectivos inteligentes, los cuales son clave para el fortalecimiento de este tipo de estrategias (figura 8).

Figura 8: Matriz de estándares básicos de competencias de lenguaje aplicados a los sistemas educativos transmedia.

\begin{tabular}{|c|c|c|c|c|c|}
\hline$\stackrel{8}{\stackrel{0}{0}}$ & \multicolumn{5}{|c|}{ DÉCIMO Y UNDÉCIMO GRADO - EDUCACIÓN MEDIA } \\
\hline \multirow{2}{*}{ 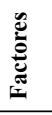 } & \multicolumn{4}{|c|}{ ENUNCIADO IDENTIFICADOR } & \multirow{2}{*}{$\begin{array}{l}\text { Colectivos } \\
\text { Inteligentes }\end{array}$} \\
\hline & Comprensión & \begin{tabular}{|l|}
$\begin{array}{l}\text { Acciones de la } \\
\text { comprensión }\end{array}$ \\
\end{tabular} & Producción & $\begin{array}{l}\text { Acciones de la } \\
\text { producción }\end{array}$ & \\
\hline \multirow{2}{*}{ 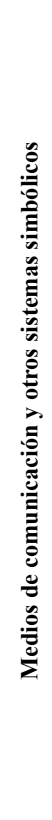 } & \multirow{2}{*}{$\begin{array}{l}\text {-Interpreto en } \\
\text { forma crítica } \\
\text { la información } \\
\text { difundida por } \\
\text { los medios de } \\
\text { comunicación } \\
\text { masiva. } \\
\text {-Retomo } \\
\text { críticamente los } \\
\text { lenguajes no } \\
\text { verbales para } \\
\text { desarrollar procesos } \\
\text { comunicativos } \\
\text { intencionados. }\end{array}$} & \multirow{2}{*}{$\begin{array}{l}\text { - Comprendo el papel } \\
\text { que cumplen los medios } \\
\text { de comunicación } \\
\text { masiva en el contexto } \\
\text { social, cultural, } \\
\text { económico y político } \\
\text { de las sociedades } \\
\text { contemporáneas. } \\
\text { - Infiero las } \\
\text { implicaciones } \\
\text { de los medios de } \\
\text { comunicación masiva } \\
\text { en la conformación de } \\
\text { los contextos sociales, } \\
\text { culturales, políticos, } \\
\text { etc., del país. } \\
\text { - Analizo los } \\
\text { mecanismos ideológicos } \\
\text { que subyacen a la } \\
\text { estructura de los medios } \\
\text { de información masiva. } \\
\text {-Asumo una posición } \\
\text { crítica frente a los } \\
\text { elementos ideológicos } \\
\text { presentes en dichos } \\
\text { medios, y analizo } \\
\text { su incidencia en la } \\
\text { sociedad actual. }\end{array}$} & $\begin{array}{l}\text { Conozco las } \\
\text { dinámicas de } \\
\text { producción de } \\
\text { sentido de los } \\
\text { diversos medios de } \\
\text { comunicación que } \\
\text { involucran diversos } \\
\text { sistemas simbólicos. }\end{array}$ & $\begin{array}{l}\text { Produzco textos } \\
\text { audiovisuales, } \\
\text { sonoros, escritos, } \\
\text { cibermediales } \\
\text { que dan cuenta de } \\
\text { mis capacidades } \\
\text { interpretativa y } \\
\text { argumentativa y } \\
\text { propositiva. }\end{array}$ & \\
\hline & & & $\begin{array}{l}\text { Identifico las } \\
\text { estructuras propias } \\
\text { de los universo } \\
\text { narrativos }\end{array}$ & $\begin{array}{l}\text { Diseño experiencias } \\
\text { aplicadas a sistemas } \\
\text { intertextuales } \\
\text { transmedia. }\end{array}$ & $\begin{array}{l}\text { Comunicadores, } \\
\text { pedagogos, } \\
\text { programadores, } \\
\text { diseñadores. }\end{array}$ \\
\hline
\end{tabular}




\begin{tabular}{|c|c|c|c|c|}
\hline 壳 & $\begin{array}{l}\text { - Comprendo e } \\
\text { interpreto textos } \\
\text { con actitud crítica } \\
\text { y capacidad } \\
\text { argumentativa. } \\
\text { - Analizo crítica } \\
\text { y creativamente } \\
\text { diferentes } \\
\text { manifestaciones } \\
\text { literarias del } \\
\text { contexto universal. }\end{array}$ & $\begin{array}{l}\text { - Elaboro hipótesis } \\
\text { de interpretación } \\
\text { atendiendo a la intención } \\
\text { comunicativa y al sentido } \\
\text { global del texto que leo. } \\
\text { - Relaciono el } \\
\text { significado de los } \\
\text { textos que leo con los } \\
\text { contextos sociales, } \\
\text { culturales y políticos } \\
\text { en los cuales se han } \\
\text { producido. } \\
\text { - Diseño un esquema } \\
\text { de interpretación, } \\
\text { teniendo en cuenta al } \\
\text { tipo de texto, tema, } \\
\text { interlocutor e intención } \\
\text { comunicativa. } \\
\text { - Construyo reseñas } \\
\text { críticas acerca de los } \\
\text { textos que leo. } \\
\text { - Asumo una actitud } \\
\text { crítica frente a los textos } \\
\text { que leo y elaboro, y } \\
\text { frente a otros tipos de } \\
\text { texto: explicativos, } \\
\text { descriptivos y narrativos. } \\
\text { - Leo textos literarios de } \\
\text { diversa índole, genero, } \\
\text { temática y origen. } \\
\text { épocas y culturas. } \\
\text { - Identifico en obras de } \\
\text { textos que leo las } \\
\text { dimensiones éticas, } \\
\text { estéticas, filosóficas. } \\
\text { el lenguaje, las } \\
\text { características formales, } \\
\text { las épocas y escuelas, } \\
\text { estilos, tendencias, } \\
\text { temáticas, géneros y } \\
\text { autores. } \\
\end{array}$ & $\begin{array}{l}\text { Produzco textos } \\
\text { argumentativos } \\
\text { que evidencian } \\
\text { mi conocimiento } \\
\text { de la lengua y el } \\
\text { control sobre el } \\
\text { uso que hago de } \\
\text { ella en contextos } \\
\text { comunicativos } \\
\text { orales y escritos. }\end{array}$ & $\begin{array}{l} \\
\\
\text { - Comprendo el } \\
\text { valor del lenguaje } \\
\text { en los procesos de } \\
\text { construcción del } \\
\text { conocimiento. } \\
\text { - Desarrollo procesos } \\
\text { de autocontrol y } \\
\text { corrección lingǘstica } \\
\text { en mi producción } \\
\text { de textos orales y } \\
\text { escritos. } \\
\text { - Caracterizo y } \\
\text { utilizo estrategias } \\
\text { descriptivas, } \\
\text { explicativas y } \\
\text { analógicas en mi } \\
\text { producción de textos } \\
\text { orales y escritos. } \\
\text { • Evidencio en mis } \\
\text { producciones textuales } \\
\text { el conocimiento de los } \\
\text { diferentes niveles de } \\
\text { la lengua y el control } \\
\text { sobre el uso que hago } \\
\text { de ellos en contextos } \\
\text { comunicativos. } \\
\text { - Produzco } \\
\text { ensayos de carácter } \\
\text { argumentativo en los } \\
\text { que desarrollo mis } \\
\text { ideas con rigor. }\end{array}$ \\
\hline
\end{tabular}

Fuente: Montoya, Vásquez y Salinas (2013), basada en MEN (2006).

\section{Conclusiones}

Ahora bien, llegados a este punto nos podríamos preguntar por la pertinencia de llevar modelos transmediales propios del mundo del entretenimiento para su aplicación 
en el ámbito educativo, más si tenemos en cuenta que ambos ámbitos se desarrollan, aparentemente, a partir de dinámicas diametralmente opuestas.

Para atender esta inquietud, encontramos diversas discusiones que resultan favorables para atender la idea de incluir lo transmedia como estrategia pedagógica. De este modo, mencionaremos como primera referencia, la indicación que Gallego hace en las conclusiones de su trabajo de maestría cuando expone la posibilidad de la aplicación de la narrativa transmedial en ámbitos educativos. Según él, esta propuesta resultaría plausible por las posibilidades de participación y co-creación que un modelo transmedial podría ofrecer.

"Por ejemplo, una clase de historia de la humanidad podría concentrarse en un período específico, el Imperio Persa, y recrear los eventos alrededor de sus protagonistas desarrollando medios para tomar decisiones de carácter político, social o militar. En un videojuego se podría recrear una campaña que requiere de la manipulación de bienes como el agua y los alimentos para sostener al ejército; el análisis de la logística permitiría al estudiante aprender sobre los principios de administración, una desatención en este aspecto llevaría a perder la guerra. Un libro podría introducir las situaciones y explorar las condiciones del contexto en la época señalada. Un cómic recrearía la mitología y su valor simbólico. La selección de las plataformas depende del objetivo educativo por alcanzar. Estos formatos podrían replicarse a otras asignaturas como matemáticas, biología, etc.” (Gallego, 2011: 63-64).

Además de la propuesta de Gallego, otra referencia en los aspectos que propenden por una idea de transmedia en entornos educativos es la de Jenkins, quien en los últimos dos años ha ampliado su propuesta de transmedia storytelling al ámbito educativo. Sostiene que en la actualidad los jóvenes, cuando se ponen en relación con los medios de comunicación, deben ser pensados como creadores de contenidos que hacen parte de lo que él llama la cultura participativa. Esta nueva cultura, puede ser definida como un fenómeno social emergente que está cautivando la atención de los jóvenes, mientras que los colegios, al no valerse de dicho fenómeno para renovar y nutrir sus programas curriculares, se están quedando claramente rezagados:

"Los programas curriculares y extracurriculares de los colegios tienen que prestar más atención al fomento de lo que llamamos la nueva alfabetización de los medios: un conjunto de competencias culturales y habilidades sociales que los jóvenes necesitan en el nuevo paisaje mediático. Casi toda la nueva alfabetización involucra habilidades sociales desarrolladas a través de la colaboración y el trabajo en red" (Jenkins, 2009: xiii) ${ }^{18}$.

18. "Schools and after-school programs must devote more attention to fostering what we call the media literacies: a set of cultural competencies and social skills that young people need in the new media landscape. The new literacies almost all involve social skills developed through collaboration and net working" (texto original). 
El mencionado autor subraya la necesidad de que los adolescentes aprendan cómo integrar el conocimiento de múltiples fuentes y medios desde su formación en los colegios. Para esto, propone el estímulo de la nueva alfabetización para desarrollar las habilidades sociales necesarias para la colaboración y el trabajo en red. Teniendo en cuenta que dichas habilidades deben ser construidas en los fundamentos de la alfabetización tradicional, las habilidades investigativas y técnicas, y la capacidad de análisis crítico que ya se enseña en las aulas de clase. De este modo, no se trata de demeritar lo que ya se hace en la enseñanza escolar, sino más bien, procurar una integración de las dinámicas propias de la cultura participativa a la estructura pedagógica y a los contenidos que se enseñan en el ámbito educativo (Jenkins, 2009).

Por otra parte, en otra obra (ápud Herr-Stephenson y otros, 2013) el autor se ocupa de reflexionar sobre la potencia que tiene la transmedialización para ser aplicada a la educación de los niños y los jóvenes. Desde su perspectiva, en la sociedad de la información, los niños aprenden jugando con la información. En este sentido, la articulación de modelos transmediales a procesos educativos fortalece el trabajo creativo de los estudiantes aumentando: la curiosidad, la exploración, la experimentación, la resolución de problemas, la especulación activa y la imaginación. Jenkins reconoce que el tema de lo transmedial articulado a la educación es ciertamente poco estudiado y, por eso mismo, sugiere este como un momento más que propicio para comenzar a explorarlo. Así, el llamado del autor es a tomarse seriamente los fenómenos de transmedialización en el ámbito educativo:

“[...] tratar de entender cómo vincular múltiples medios para crear nuevas experiencias pedagógicas, estar listos para jugar juntos alrededor de los materiales de una franquicia transmedial, invitar a los niños a explorar lo que significa leer una historia a través de las fronteras y los límites entre diferentes textos y diferentes medios de comunicación" (Herr-Stephenson y otros, 2013: 9) $)^{19}$.

Vemos entonces que, siguiendo lo propuesto por Jenkins, la aplicación de modelos transmediales al contexto educativo no es solo coherente, sino también necesaria. La puesta en juego de la cultura participativa que tanto cautiva a los jóvenes, sumada a la introducción de nuevas tecnologías de la información y al estancamiento de los contenidos pedagógicos en los colegios, hace que estemos en un momento ideal para explorar las múltiples posibilidades que pueden abrirse con la articulación de modelos transmediales, como el que hemos propuesto, al ámbito de la educación.

En síntesis, con esta propuesta de transmedialización de La Odisea como estrategia pedagógica para incentivar la lectura, retomamos y ampliamos las ideas esbozadas por Gallego, al igual que asumimos los retos dejados por Jenkins al compartir con él la

19. " [...] to try understand how to link multiple media together to create new pedagogical experiences, to be ready to play together around the materials of a transmedia franchise, to invite children to explore what it means to read a story across the borders and boundaries between different texts and different media" (texto original). 
inquietud de sabernos en un momento coyuntural en el que la reflexión sobre los fenómenos transmediales debe comenzar a trascender los marcos establecidos por el mundo del espectáculo y del entretenimiento, para explorar sus potencialidades en otros ámbitos en donde pueden resultar no solo interesantes, sino en definitiva, necesarias.

\section{Fuentes consultadas}

Appadurai, A. (2001). La modernidad desbordada. Buenos Aires: Trilce.

Departamento Administrativo Nacional de Estadística, DANE (2013, abril 23). "Encuesta de consumo cultural. Año 2012”. Extraída el 2/II/2015 desde http://www.dane.gov.co/files/ investigaciones/eccultulral/presentacion_ecc_2012.pdf

Eco, U. (1993). Lector in fábula. La cooperación interpretativa en el texto narrativo. Barcelona: Lumen.

Gallego, A. (2011). Diseño de narrativas transmediáticas. Guía de referencia para las industrias creativas de países emergentes en el contexto de la cibercultura. Manizales: Universidad de Caldas.

Genette, G. (1989). Palimpsestos. La literatura en segundo grado. Madrid: Taurus.

Gosciola, V. (2012). "Narrativas Transmídia: Conceituaçâo e origens”. En Campalans, C.; Renó, D., y Gosciola, V. (eds.) Narrativas transmedia. Entre teorías y prácticas. Bogotá: Universidad del Rosario.

Gray, Jonathan (2010). Show sold separately: promos, spoilers, and other media paratexts. Nueva York: New York University.

Hernández Pérez, M. y Grandío Pérez, M. (2011). "Narrativa crossmedia en el discurso televisivo de Ciencia Ficción. Estudio de Battlestar Galactica (2003-2010)". Área Abierta, núm. 28. Extraída el 30/I/2015 desde http://revistas.ucm.es/index.php/ARAB/article/view/ ARAB1111130004A/4031

Herr-Stephenson, B. y otros (2013). "T is for transmedia: Learning through transmedia play". Extraída el 30/I/2015 desde http://www.annenberglab.com/ projects/t-transmedia

Jenkins, $\mathrm{H}$.

_(2009). Confronting the Challenges of Participatory Culture. Education for the 21st Century. Cambridge: Massachusetts Institute of Technology

_(2008). Convergence Culture. La cultura de la convergencia de los medios de comunicación. Barcelona: Paidós.

_(2003, enero 15). “Transmedia Storytelling”. Extraída el 30/I/2015 desde http://www. 
technologyreview.com/news/401760/transmedia-storytelling/

_(2001, junio 1). “Convergence? I Diverge”. Extraída el 30/I/2015 desde http://www. technologyreview.com/article/401042/convergence-i-diverge/

Kinder, M. (1991). Playing with Power in Movies, Television, and Video Games: From Muppet Babies to Teenage Mutant Ninja Turtles. Berkeley: University of California Press.

Kirkman, R.; Adlard, C.; Moore, T. y Rathburn, C. (2013). The Walking Dead Compendium (volumen 1). California: Image Comics.

Kirkman, R.; Adlard, C. y Rathburn, C. (2013). The Walking Dead Compendium (volumen 2). California: Image Comics.

Klastrup, L. y Tosca, S. (2004). "Transmedia World. Rethinking Cyberworld Design" [conferencia]. CW '04 Proceedings of the 2004 International Conference on Cyberworlds. Washington D. C.: IEEE Computer Society.

Lévy, P. (2004). Inteligencia Colectiva: Por una antropología del ciberespacio. Washington: Organización Panamericana de la Salud.

Long, G. (2007). Transmedia Storytelling. Business, Aesthetics and Production at the Jim Henson Company. Massachusetts: MIT.

Matos, D. y Vicente, A. (2011). The Walking Dead: Caminando entre los muertos. Palma de Mallorca: Pretextos Dolmen.

Ministerio de Educación Nacional, MEN (2006). Estándares básicos de competencias de lenguaje. Bogotá: Editor Ministerio de Educación Nacional.

Montoya, D. (2014). "La ficción Zombi: Una mirada transmedia". XII Congreso Latinoamericano de Investigadores de la Comunicación. Lima: PUCP

Montoya, D.; Vásquez, M. y Salinas, H. (2013). "Sistemas intertextuales transmedia: Exploraciones conceptuales y aproximaciones investigativas". Co-herencia, vol. 9, núm. 18, pp. 137-159.

Odisea. Una Narrativa Transmedia (2013). "Inicio”. Extraída el 2/II/2015 desde http:// odiseaunanarrativatransmedial.tk/

Plan Digital TESO (2013). "Semilleros de investigación. Transmedia educativa. Transmedia, generando nuevos mundos y aprendizajes". Extraída el 2/II/2015 desde http:// www.planteso.edu.co/hometeso/semilleros-de-investigacion/transmedia/

Pratten, R. (2011). "Getting started in transmedia storytelling. A practical guide for beginners". Extraída el 30/I/2015 desde http://www.slideshare.net/ZenFilms/getting- 
started-in-transmedia-storytelling?from $=$ embed

Scolari, C.

_(2010). "Narrativa transmediática, estrategias cross-media e hipertelevisión”, pp. 69-100. En Scolari, C.; Piscitelli, A. y Maguregui, C. (eds.). Lostología. Estrategias para entrar y salir de la isla. Buenos Aires: Cinema.

_(2009). "Transmedia storytelling. Implicit consumers, narrative worlds, and branding in contemporary media production". International Journal of Communication, núm. 3, pp. 586-606. Extraída el 30/I/2015 desde http://beta.upc.edu.pe/matematica/portafolios/ nmynt/ transmedia_storytelling-.pdf

Varela, M. (1999). "De las culturas populares a las comunidades interpretativas". Extraída el 30/I/2015 desde http://www.periodismo.uchile.cl/talleres/teoriacomunicacion/archivos/ varela. pdf 